\begin{tabular}{|c|l|}
\hline Title & $\begin{array}{l}\text { STUDIES OF THE HYDROGEN ELECTRODE PROCESS ON LEA D CA THODE AT VERY HIGH ELECTRODE } \\
\text { POTENTIALS }\end{array}$ \\
\hline Author(s) & MITUYA, A tusi; Y A MAZA KI, Tsunehiro \\
\hline Citation & JOURNAL OF THE RESEARCH INSTITUTE FOR CA TALY SIS HOKKA IDO UNIVERSITY, 7(1), 42-51 \\
\hline Issue Date & 1959-09 \\
\hline Doc URL & http://hdl.handle.net/2115/24692 \\
\hline Type & bulletin (article) \\
\hline File Information & $7(1) \_P 42-51 . p d f$ \\
\hline
\end{tabular}

Instructions for use 


\title{
STUDIES OF THE HYDROGEN ELECTRODE PROCESS ON LEAD CATHODE AT VERY HIGH ELECTRODE POTENTIALS
}

By

\author{
Atusi Mrtuya and Tsunehiro Yamazaki*) \\ (Received July 26, 1959)
}

Number of works have been reported on the hydrogen electrode of lead, but they deal exclusively with higher cathodic polarization above $600 \mathrm{mV}$ and, besides, their results disagree with each other as those of Hicking and SAIT' ${ }^{1)}$, Bockris ${ }^{2)}$, and Kavanov and Jora ${ }^{3)}$ as shown in Fig. 1. More or less similar situation holds with the works on other metals of hydrogen electrode.

Nevertheless, it is often tried to extrapolate so-called exchange current $i_{0}$ at zero polarization, as an important criterion for the mechanism of hydrogen electrode, from cathodic current $i$ observed at high cathodic polarization relying upon the linear relation between $\log i$ and cathodic polarization $-\eta$.

One of the authors ${ }^{4}$ has previously reported his work on a mercury cathode at very low cathodic polarization by preparing and operating the electrolytic cell exclusively in vacuum, and has showed the presence of a break in the linear $\eta-\log i$ relation. This break is just that predicted by the dual theory ${ }^{5)}$ indicating that the above drastic extrapolation is of little sense; experiments must be extended to the region of lower cathodic polarization for elucidating the mechanism of hydrogen electrode reaction.

The present authors have conducted the experiments on lead cathode at expremely low cathodic polarization as reported below, by vacuum process as in the previous experiment on mercury cathode ${ }^{4}$.

\section{Experimental}

Materials were prepared as follows:

*) A. M. and T. Y.: Department of Chemistry, St. Paul's University (Rikkyo Daigaku), Ikebukuro, Tokyo. 


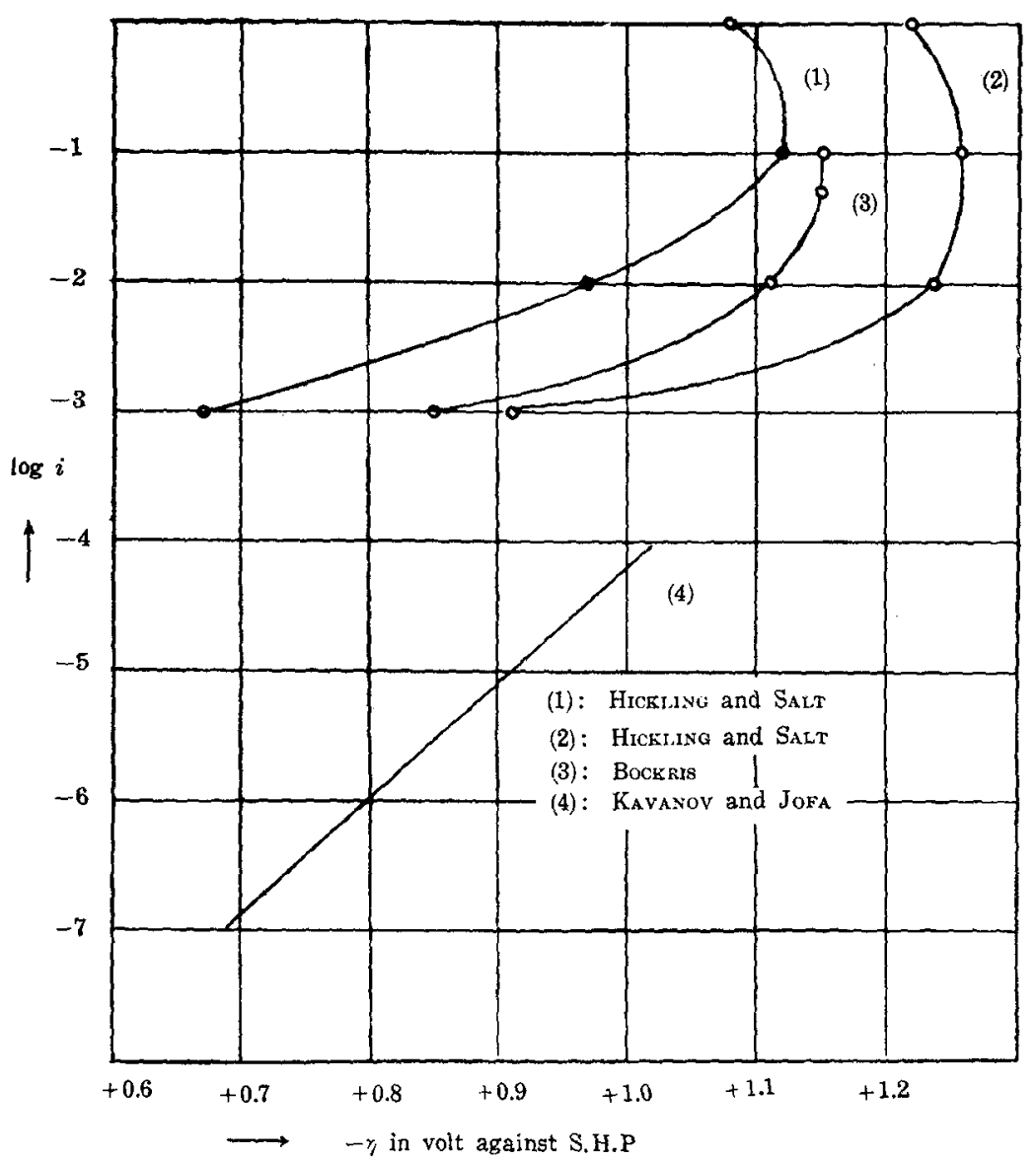

Fig. 1. Hydrogen overvol tage on lead cathode, observed by several workers.

(1) Pb-cathode: Lead metal of purity $99.996 \%$, guaranteed by the Mitui Metal and Mining Co., was melted at $400^{\circ} \mathrm{C}$ in compartment A in Fig. 2 under high vacuum (below $10^{-5} \mathrm{mmHg}$ ). Line $\mathrm{S}_{1}$ was now sealed off and hydrogen gas, purified by passing it through palladium thimble, was slowly introduced through a breakable joint in the Figure to push the meit from $A$ to $C$, through the trap $B$ where the oxides in the bulk of the lead metal was separated. A glass tube D with a platinum contact sealed in a glass rod was thus covered with the pure molten lead by its lower end as shown in the Figure, and hydrogen gas filled now the combined spaces of $A, B$ and $C$ up to the greeseless $\operatorname{tap} T$. As the lead cooled and solidified, it plucked the inner tube 


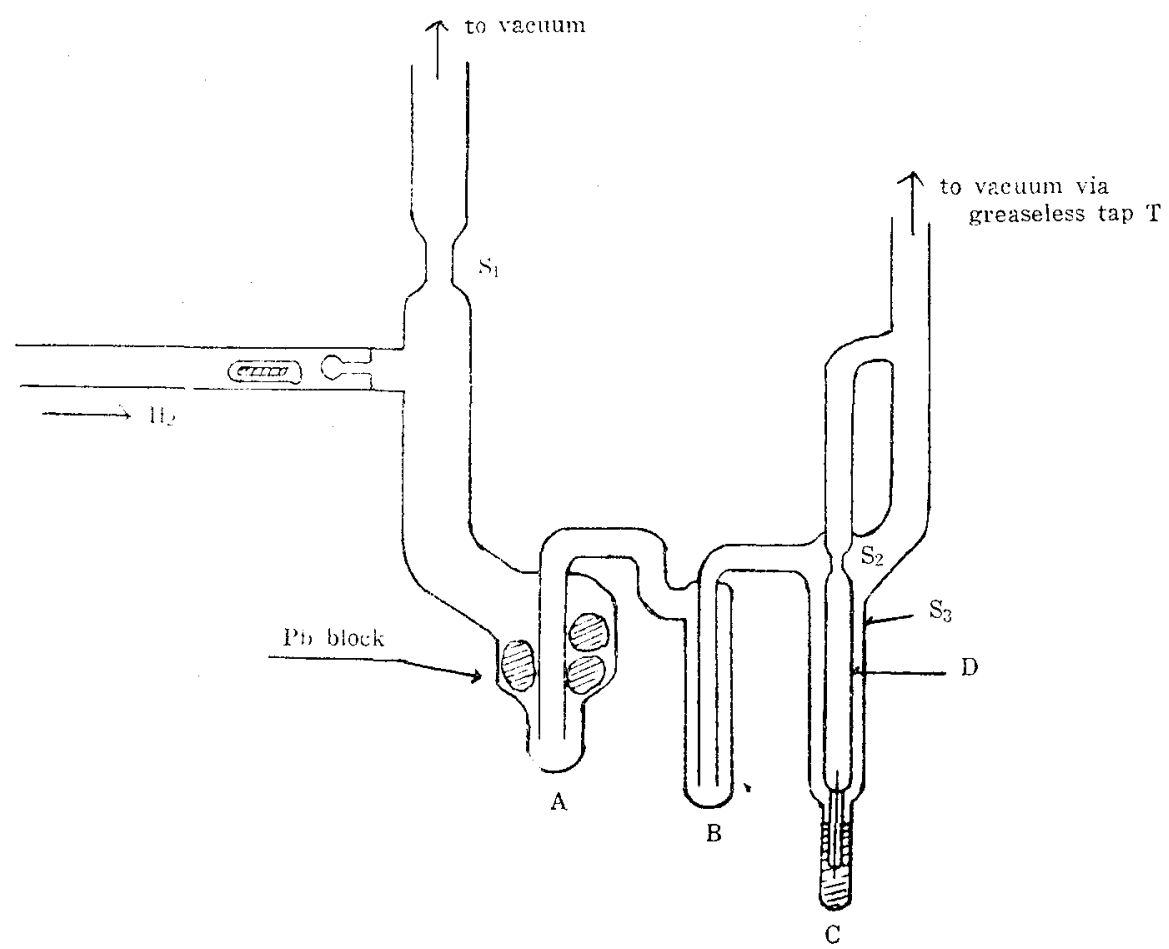

Fig, 2. Scheme of preparation of cathode materials.

at $S_{2}$, where a scratch is preliminarily filed, keeping the geometry of the lead cathode and the platinum seal as shown in the Figure.

The outer tube was now cut off at $S_{3}$ and glass around the lead electrode was removed by hydrogen fluoride solution in air. The lead netal thus obtained had a color and brilliant shine like a polished silver and firmly fixed to the platinum contact. The cathode thus prepared was now worked out to make Cell-I, as shown in Fig. 3, and subjected to cathodic purification of the lead electrode in electrolytically purified $0.1 \mathrm{~N}-\mathrm{HCl}$ solution in $\mathrm{F}_{2}^{\mathrm{b}}$ (cf. (III)) under vacuum.

The cathodic purifiration was conducted similariy as in the previous work $^{4} *$ using redistilled water in $\mathrm{F}_{3}$ for wash and $\mathrm{F}_{4}$ for drain. After these procedures, the constrictions $S_{4}, S_{5}$ and $S_{6}$ were sealed off, and a proper amount of electrolytically purified $0.1 \mathrm{~N}-\mathrm{HCl}$ solution in $\mathrm{F}_{2}^{\mathrm{a}}$ (cf. (III)) was introduced by a careful distillation through the breakable joint $a$, and then the purified hydrogen was introduced through $b$, just

*) Cf. Ref. $4, \$ 1,(1)$. 
The Hydrogen Electrode Process on Lead Cathode

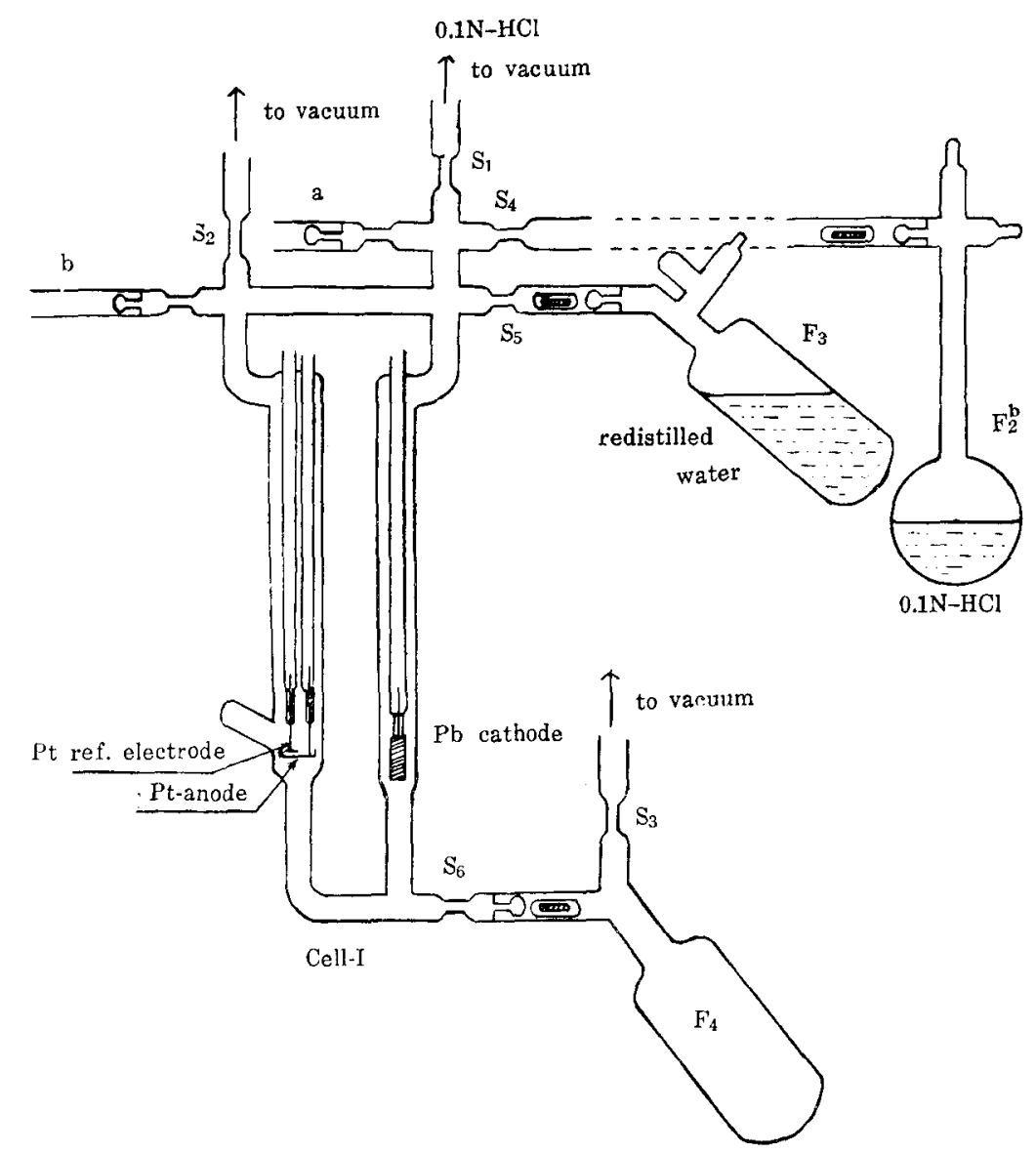

Fig. 3. Dia ram of the pre-electrolitic purification apparatus for the electrodes in electrolytically purified.

to put the gas-liquid interface at the platinum reference electrode in order to facilitate it to work as a reversible hydrogen electrode. The cell was completed by sealing off the constrictions a and b. Its complete make-up is shown in Fig. 4. The apparent area of the cathode was $5.8 \mathrm{~cm}^{2}$ and the hydrogen gas pressure was $560 \mathrm{~mm} \mathrm{Hg}$ at $19^{\circ} \mathrm{C}$.

(II) Pt-electrodes: Because of very large apparent area of the cathode, a platinum anode of the form of a shallow dish as shown in Figs. 3 and 4 was used to prevent any appreciable polarization. The platinum reference electrode was a platinized platinum plate, which was placed close at the bottom of the dish in order to prevent any granule of platinum black from coming down into the solution. These platinum 


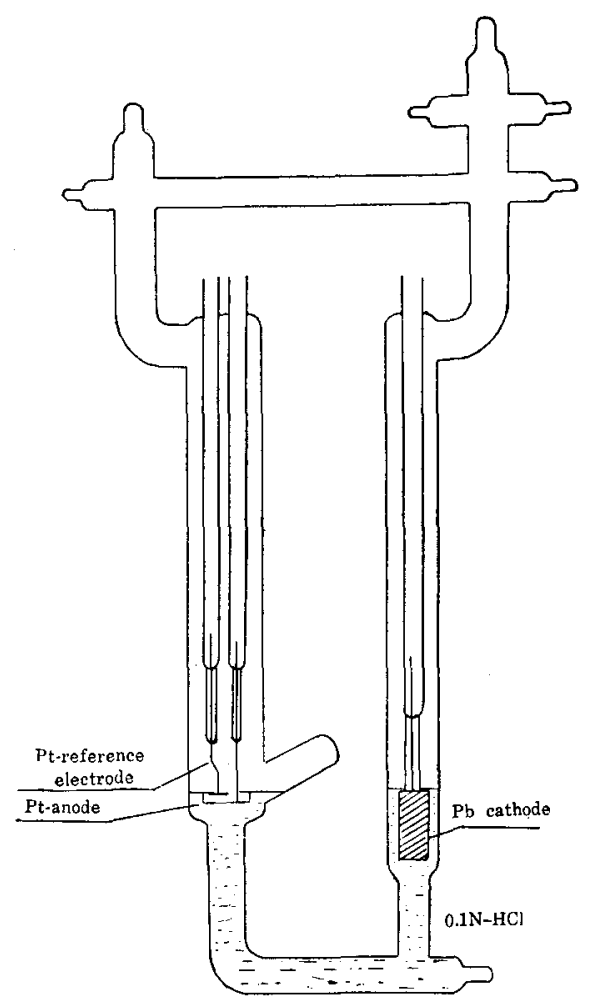

Fig. 4. Electrolytic cell.

electrodes were platinized in this dish to cover the whole surface of the plate and the inner surface of the dish only. The methods of the purification of the platinum electrodes were the same as those described in the previous report ${ }^{4}$.

(III) $\mathrm{H}_{2}$ gas and $0.1 \mathrm{~N}-\mathrm{HCl}$ solution: Cylinder hydrogen was purified by passing it through a palladium thimble, a liquid oxygen trap, being kept afterwards from contacting any greased tap or joint.

The pre-electrolytic purification of $0.1 \mathrm{~N}-\mathrm{HCl}$ solution was carried out in Cell-II under vacuum, as shown in Fig. 5. Solution in flask $\mathrm{F}_{1}$ was introduced into Cell-II by vacuum distillation, and the electrolysis was continued for 5 days, applying a terminal voltage of $0.4 \mathrm{~V}$ between platinum anode and lead cathode. After the electrolysis, the solution was introduced into evacuated flask $F_{2}^{a}$ and $F_{2}^{b}$ via $S_{3}$, breaking the joint by an impact. The amounts of solution in $F_{2}^{a}$ and $F_{2}^{b}$ were so adjusted that they just touched the surface of the platinum reference 


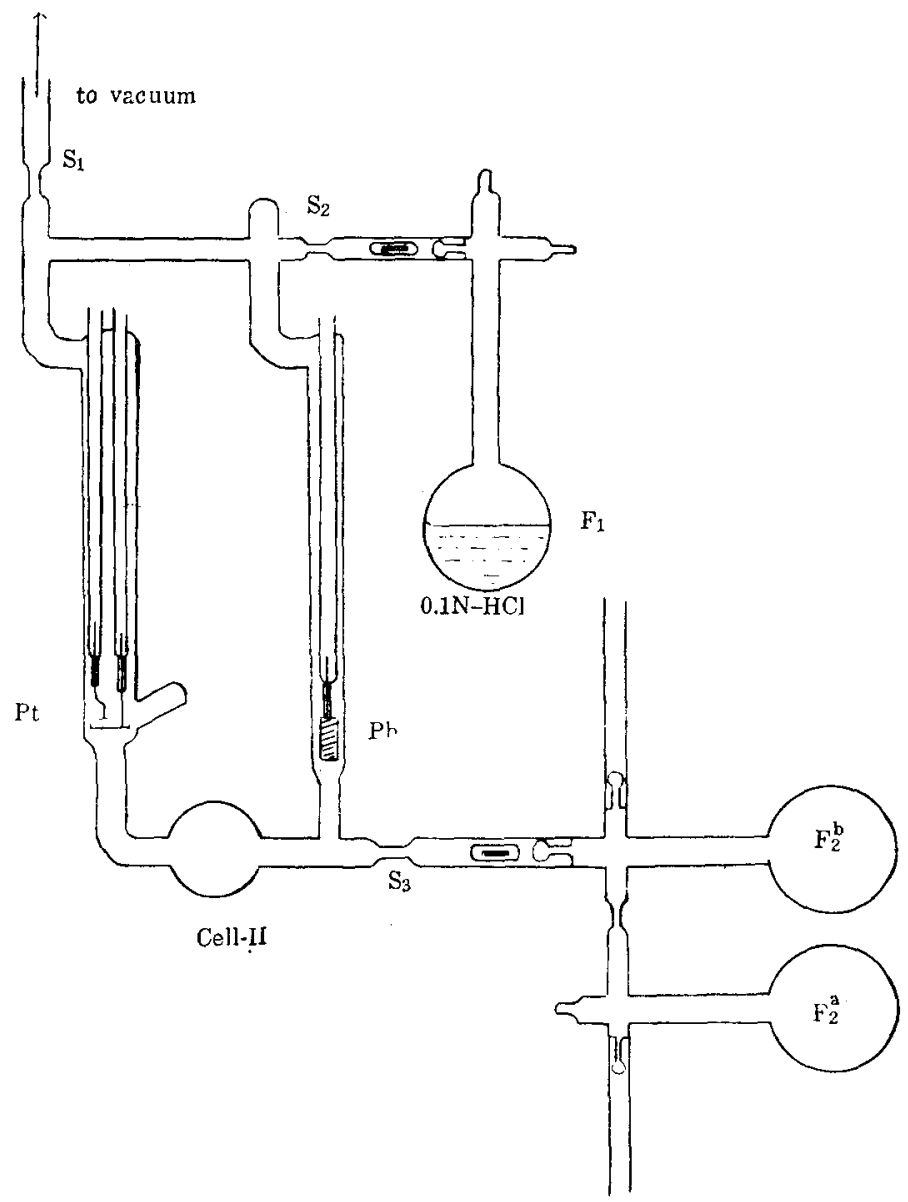

Fig. 5. Diagram of the pre-electrolytic purification apparatus for $0.1 \mathrm{~N}-\mathrm{HCl}$.

electrode when introduced into Cell-I for completing the cell as described in (I) or into Cell-II for pre-electrolysis respectively.

The measurement of the current density at each fixed polarization of the lead cathode was conducted as follows:

When the cathode was polarized at a constant cathodic polarization after being left unpolarized, the current $i$ decreased with progress of time to tend asymptotically to a definite value particular to the applied polarization $\eta$. Whenever the cathodic polarization was increased (decreased) from a constant value at which an asymptotic current was approached as above, the current increased (decreased) first instan- 
taneously and then slowly decreased (increased) to tend to an asymptotic value higher (lower) than the foregoing one. The asymptotic current at any definite polarization was almost reproducible as in the case of mercury ${ }^{4}$. The time required for approaching the asymptotic current to a certain extent varied from 20 to $300 \mathrm{hrs}$ according as the cathodic polarization was higher or lower. It was concluded from these experimental facts similarly as in the previous case ${ }^{4)}$ that the asymptotic value gives the current of genuine hydrogen electrode reaction at the appropriate constant cathodic polarization, where the ionization of metal attains partially equilibrium.

The polarization of the anode referred to the reference electrode was completely immeasurable for the asymptotic current density below $2 \times 10^{-i} \mathrm{amp} . \mathrm{cm}^{-2}$. Results of measurements at such experimental condition were only adopted as sound and shown in Fig. 6, since then any measurable potential gradient in the solution may be absent.

\section{Result and Discussion}

Fig. 6 shows the observed $\eta-\log i$ relation ${ }^{*)}$, where annexed numbers to points denote their order of the measurements. Since $i$ at a constant polarization should approach the asymptotic value from upwards or downwards according as the constant cathodic polarization is raised or lowered from the foregoing constant one, the points in Fig. 6 consistently determines the $\eta-\log i$ curve as drawn in the figure, which is reliable at least down to point 6 .

We note now that the unidirectional backward current $\hat{i}$ is practically ignorable compared with the unidirectional forward current $\vec{i}$ of the hydrogen electrode reaction

$$
2 \mathrm{H}^{+}+2 \mathrm{e}=\mathrm{H}_{2}
$$

in the region of the present measurement, according to the equation $i=\vec{i}\left(1-\exp \frac{2 F_{\eta}}{\nu R T}\right)^{6) 7}$, whether the stoichiometric number $\nu$ of the ratedetermining step be 1 or 2 . It follows that Fig. 6 practically presents $\eta-\log \vec{i}$ curve and moreover that the latter is by no means linear.

* This value may be referred to the standard hydrogen electrode which is calculated by adding $\frac{R T}{2 F} \cdot \log \left(\frac{P}{760} \cdot \frac{1}{C}\right)$ to the directly observed value referred to the reversible hydrogen electrode in the cell, where $P$ is the hydrogen pressure and $C$ is the activity of the $\mathrm{H}^{+}$ions in the solution. 


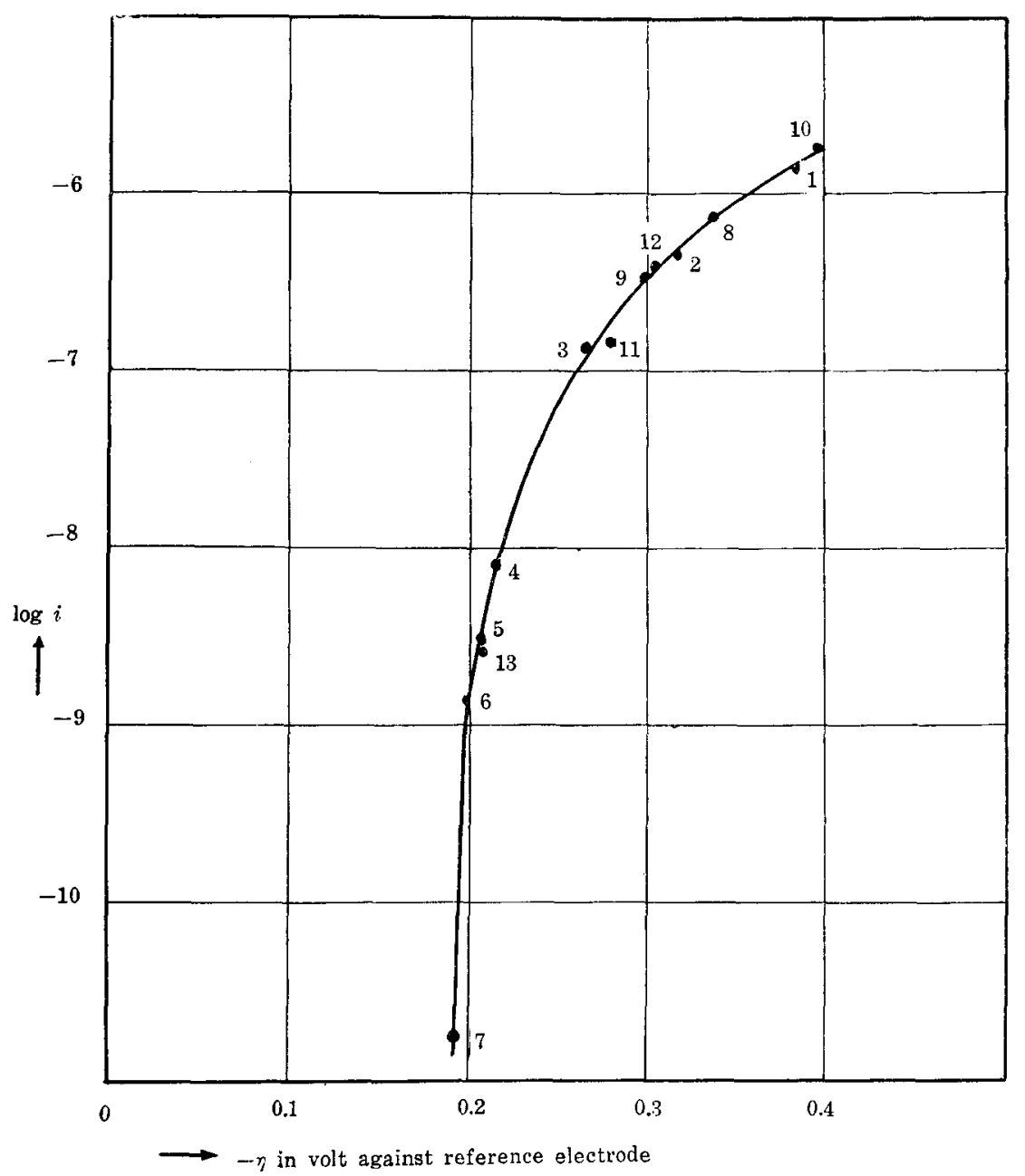

Fig. 6. The $n-\log i$ relationship of lead hydrogen electrode.

The usual linear extrapolation of $i_{0}$ from measurements of $i$ at higher cathodic polarization is in consequence of little sense.

It was found that at zero current the potential of the cathode rose slowly up to $-0.186 \mathrm{mV}$ within $24 \mathrm{hrs}$, which kept perceptibly constant for $400 \mathrm{hrs}$ as observed after the series of measurements shown in Fig. 6 in contrast with in the case of hydrogen electrode of mercury, when the cathode potential attained practically to that of reversible hydrogen electrode at zero current. This result could be understood on the ground of the observed rapid decrease of $i$ with decreasing 
cathodic polarization as follows. The metal ion concentration at the equilibrium of the ionization of the metal decreases with increasing cathodic polarization. The concentration is hence reduced during the series of the measurement to the value corresponding to the cathodic polarization greater than $c a .200 \mathrm{mV}$. The metal electrode should now be ionized a good deal in order to attain the equilibrium concentration at the reversible hydrogen electrode potential. The positive electricity required for the metal ionization must, however, be supplied by the progress of the hydrogen electrode reaction. But since the latter is extremely slow at lower cathodic polarization, the metal ionization is practically checked as observed. It might be pointed out that the observed extremely slow hydrogen electrode reaction at lower cathodic polarization favours the inhibition of self-discharge of lead accumlators.

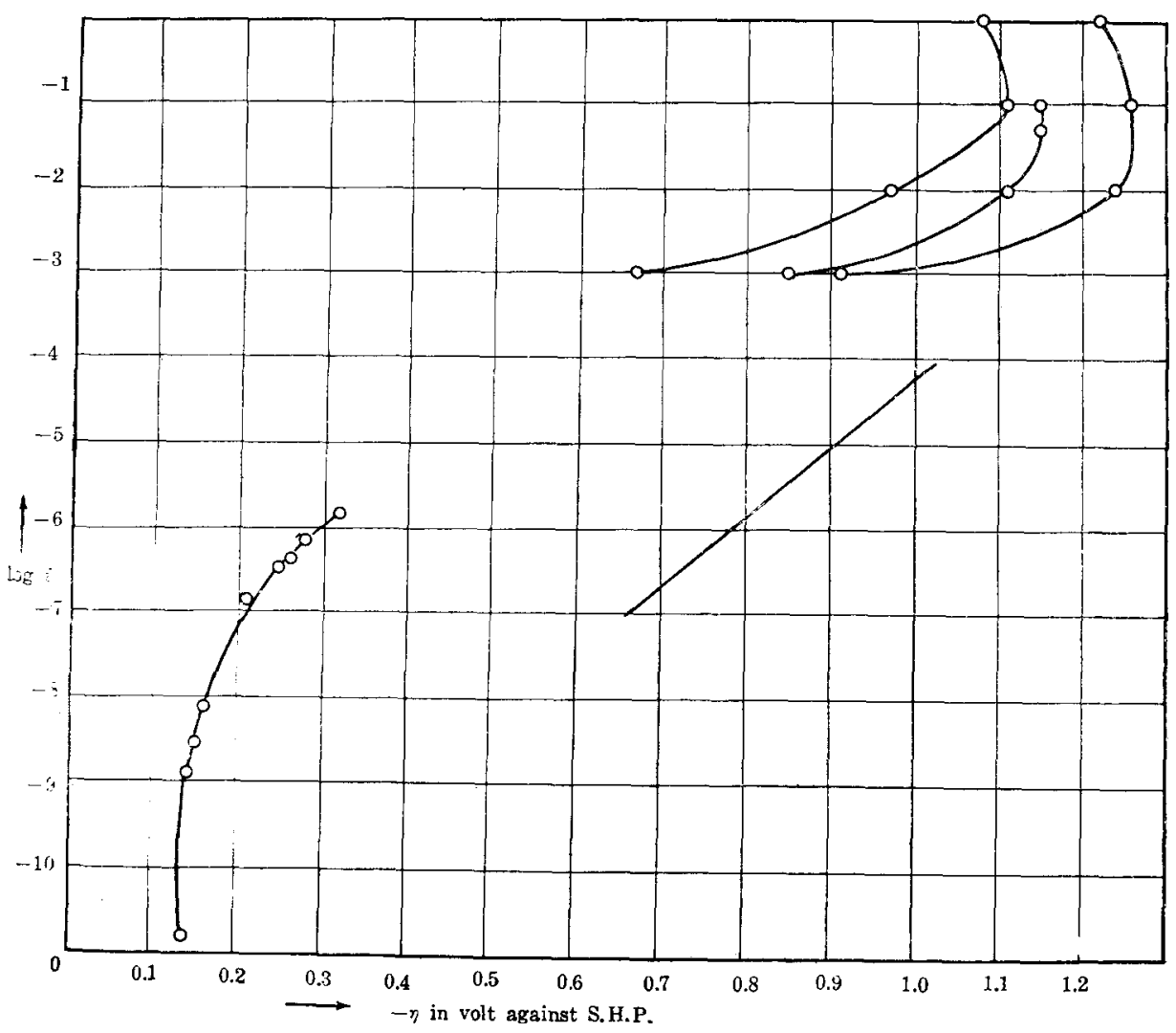

Fig. 7. Comparison of the present $\eta-\log i$ relation with earlier results. 
Fig. 7 compares the present results with earlier ones mentioned above $^{122) 3}$. No description is found in these earlier works except in that of Kavanov and $\mathrm{JoFA}^{3)}$ about the measurement of the reference electrode for measuring the cathodic polarization.

\section{Summary}

Cathodic current $i$ of the hydrogen electrode process on lead cathod was observed over the range of cathodic polarization from 0.18 to 0.39 volt by vacuum operation. The $\log i$ was found not linear function of $\eta$ and to decrease with increase of $\eta$ more rapidly at highər $\eta$ just as in the case of mercury cathode (Ref. 4). Linear extrapolation of $\log i$ to $\eta=0$ for the exchange current $i_{0}$ from higher cathodic polarization is in consequence quite impracticable.

\section{Acknowledgement}

The authors express their deepest thanks to Prof. J. HoRIuTI of Hokkaido Univ. for his encouragement and kind advice.

\section{References}

1) A. Hickling and F. W. Salt, Trans. Faraday Soc. 36, 1226 (1940).

2) J. O'M. BOCKRIS, Trans. Faraday Soc. 43, 417 (1947).

3) B. KaVANOV and Z. A. Jofa, Acta Physicochim. U.R.S.S. 10, 617 (i939).

4) A. MrTUYA, J. Res. Inst. Catalysis, Hokkaido Univ. 4, 228 (1957).

5) J. Horiuti, T. KeII and K. Hirota, J. Res. Inst. Catalysis, Hokkaido Univ. 2, 1 (1951).

6) J. Horiuti and M. IKushima, Proc. Imp. Acad. Tokyo 15, 39 (1939).

7) J. HoRIuti, J. Res. Inst. Catalysis Hokkaido Univ. 1, 8 (1948). 